\title{
Petite encyclopédie de la reproduction biologiquement correcte (PERBC)
}

\author{
Jean-Claude Kaplan
}

Les caractères gras renvoient aux entrées.

Accouchement. Arch., anim., biol. incorr.: Chez Homo sapiens sapiens enfantement à la mode animale au terme d'une gestation intra-utérine de 9 mois. Acte dangereux pour la mère et l'enfant, maintenant supplanté par la PTM (voir aussi Homo sapiens mitoticus). Non remboursé par la Sécurité Sociale, sauf cas particuliers (v. Autoclone et autogestation).

A.D. Anglais: 1. Ring.: Anno Domini (c'est-à-dire après Jésus-Christ). 2. Mod.: After Dolly (c'est-à-dire à partir de 1997, début de l'ère post darwinienne).

Adam. Premier donneur de noyau diploïde pour clonage. V. Ève.

Adoption. 1. Ring. et Biol. incorr.: A. d'enfant: procédé par lequel les couples stériles se procuraient un enfant sans même en connaître le génotype 2. Mod. et Biol. corr.: A. de clone: mode d'adoption de l'ère post-darwinienne, utilisé lorsque, pour une raison quelconque, le noyau ne provient d'aucun des parents. Le noyau doit résulter d'un don anonyme inscrit au registre des Copyright (voir DDN).

Autoclone. Cas particulier d'homoclone où le noyau diploïde et l'ovocyte proviennent de la même femme.

Autogestation. GIV après implantation d'un autoclone dans l'utérus de la femme qui a fourni à la fois le noyau somatique et l'ovocyte dénoyauté.

Barrière d'espèce. Encore un tabou 670 reproduction asexuée a permis de s'en affranchir.

BD. Anglais : Before Dolly, c'est-à-dire avant 1997.

Bioéthique. Code du biologiquement correct. Malléable sous la pression sociale. Exemple: le clonage humain est devenue licite sous la pression des couples stériles.

BPTM. Biotech PTM. Désigne les entreprises privées assurant la prestation des PTM, notamment la GEV. Conventionnées si conformes à la norme Bio Iso 9001. Très menacées par les projets d'introduction sur le marché de kits (cf. publicité sur le web: click ' $n$ ' clone).

CLHCC. Central London Hatchery and Conditioning Centre: première structure de GEV imaginée par A. Huxley dès 1932 dans Brave New World. Ancêtre des BPTM.

Clonage humain. Variété de clonage cellulaire permettant d'obtenir un individu entier en réveillant la totipotentialité latente du noyau de n'importe quelle cellule somatique diploïde par immersion dans un ovocyte nulloploïde (voir zyclone). Procr. : nouveau mode post-darwinien de reproduction asexuée permettant d'obtenir la copie conforme d'un individu à partir d'un noyau provenant de n'importe quelle cellule somatique, donc d'éviter les incertitudes de la loterie méiotique. Soc.: Innovation beaucoup mieux accueillie par la société post-moderne que le clonage moléculaire («manipulations génétiques»). A permis de créer Homo sapiens mitoticus. Philos.: A permis de s'affranchir du hasard et de la nécessité. Droit: Si l'individu est une copie conforme du donneur de noyau il possède néanmoins une âme distincte et en diffère autant que s'il s'agissait d'un jumeau monozygote élevé à part («l'homme ne se résume pas à son génome»). Voir Individu cloné.

Clone. Jumeau monozygote du donneur de noyau, aux mitochondries près. Voir aussi Individu cloné.

Comité d'éthique. 1. Ring.: assemblée de sages chargés de préciser la frontière entre l'acceptable et l'inacceptable en matière de biologie. 2. Mod.: assemblée de sages chargés de préciser quand et comment l'on peut céder à la pression sociale. Compte tenu de l'évolution rapide des mentalités et des progrès de la science, les comités sont consultés de plus en plus souvent. A présent ils siégent en permanence (24h sur 24 ), et statuent directement sur le web.

Coup de foudre. 1. Ring.: amour subit. 2. Mod.: bouffée de phéromones. 3. Procr.: électroporation permettant la pénétration d'un noyau diploïde dans un ovocyte nulloploïde.

CUCH. Convention Universelle des Copyright de l'Homme, en remplacement de la Déclaration Universelle des Droits de l'Homme.

Darwin. La reproduction asexuée a enfin permis de s'en débarrasser.

DDN. Don de noyau somatique diploïde à des fins de clonage, si aucun des parents ne peut ou ne veut être cloné. Doit satisfaire aux critères de la CUCH. 
DDO. Don d'ovocyte dénoyauté (nulloploïde) à des fins de clonage. Doit satisfaire aux critères de la CUCH.

Dionysos. Myth.: fruit d'une reproduction sexuée, avec gestation extrautérine (intracrurale) chez le père (Jupiter).

Dolly. Mieux qu'Adam et Ève.

Don de noyau. Voir DDN.

Don d'ovocyte. Voir DDO.

Droits de l'Homme. Remplacés par ceux du Copyright. V. CUCH.

Enfant. 1. Ring.: fruit aléatoire d'une reproduction sexuée, donc de génotype et de phénotype imprévisibles (H. sapiens sapiens). 2. Mod.: clone post-darwinien protégé par Copyright (H. sapiens mitoticus).

Électroporation. Voir Coup de foudre.

Ève. Premier individu créé par clonage à partir de cellules somatiques diploïdes. L'opération a entraîné une perte du chromosome $\mathrm{Y}$ et une unidisomie du chromosome X (v. Adam).

Famille nucléaire. 1. Arch.: couple et ses enfants résultant d'une PS. 2. Mod.: couple et ses enfants résultant d'une transplantation nucléaire.

Gamète. 1. Arch.: précurseur haploïde et sexué (mâle $=$ spermatozoïde. femelle $=$ ovocyte) nécessaire à la conception d'un zygote. Soumis à tous les aléas de la loterie méiotique. 2. Mod.: ovocyte dénoyauté, seul dérivé gamétique (nulloploïde) nécessaire à la PTM.

GIV. Gestation in vivo. Biol. incorr.: 1. Intra-utérine, soit chez la femme ayant produit l'ovocyte, soit dans un utérus de location; 2. Extra-utérine, le plus souvent chez un homme, soit péritonéale, soit intracrurale (v. Dionysos).

GEV. Gestation ex vivo. Biol. corr.: seule technique remboursée par la Sécurité sociale si elle fait partie d'un protocole de PTM. Obligatoirement effectuée dans une BPTM.

$\mathrm{m} / \mathrm{s} n^{\circ} 5$, vol. 13 , mai 97
Grossesse. Arch., Ring., Biol. incorr.: gestation intra-utérine d'un embryon conçu selon un protocole de reproduction sexuée (v. Accouchement).

Homo sapiens mitoticus. Biol. corr. : primate délivré de son animalité, dérivant de $H$. sapiens sapiens. Espèce définitivement fixée grâce au clonage, qui a permis de s'affranchir du hasard et de la nécessité (voir Homo sapiens sapiens).

Homo sapiens sapiens. Arch.: stade primitif de l'espèce humaine, esclave de la logique méiotique. Synonyme de H. sapiens meioticus. Ancêtre de Homo sapiens mitoticus.

Homoclone. Clone dont le noyau donneur et l'ovocyte receveur sont de la même espèce.

Inacceptable. Demain acceptable.

Individu post-coïtal. Arch., Ring., Biol. Incorr.: Individu conçu et enfanté à l'ancienne (v. Homo sapiens sapiens).

Individu cloné (ou clone). Individu pouvant servir, soit à l'accomplissement d'un projet parental (enfant baptisable), soit de réservoir de cellules ou d'organes parfaitement biocompatibles dont on peut avoir besoin sa vie durant (organisme brevetable). Jurisprudence : ces deux destinées sont mutuellement exclusives; le choix doit être fait dès la naissance et est irrévocable. Un IC ne peut être à la fois baptisé et breveté.

Inné et acquis. Vieux débat (en anglais nature vs nurture) impossible à trancher tant que l'on ne disposait d'aucun moyen expérimental statistiquement satisfaisant. A la fin du XX $\mathrm{XX}^{\mathrm{e}}$ siècle l'avènement de la reproduction asexuée par clonage a enfin permis de quantifier précisément la part de l'inné et de l'acquis. L'expérience décisive a consisté à comparer, en les plaçant dès leur naissance dans des environnements variés, 20 clones de Groseille et 20 clones de Le Quesnoy (cf. E. Chatiliez. La vie est un long fleuve tranquille, 1988, Fr., 35 mm, coul., 90 mn).

Interdit biologique. Ne concerne que les expériences qui sont aujourd'hui théoriquement impossibles et biologiquement irréalisables.

Libération de la femme. Obtenue par la PTM.

Maïeutique. Philos., Procr.: elle est bien morte, comme la Méiotique.

Méiotique. Biol. incorr.: loterie de la sexualité.

Mentalités. Heureusement elles changent, ce qui donne du travail aux comités d'éthique.

Mère mitochondriale. Donneuse d'ovocyte dénoyauté, donc nulloploïde (v. DDO).

Mère nucléaire. Donneuse de noyau diploïde (v. DDN)

Moratoire. Prélude à l'acceptation par la société d'une pratique biologique très innovante, et considérée comme inacceptable jusqu'à ce qu'elle devienne techniquement réalisable. Ex. : le clonage humain.

Mutations. L'épée de Damoclès de la reproduction sexuée. Les négliger complètement quand il s'agit de reproduction asexuée par clonage.

Onzième commandement. "Tu ne cloneras point» (en anglais: "Thou shalt not clone»). Édicté pour être immédiatement outrepassé, comme les Dix premiers.

Ovocyte. 1. Arch.: O. nucléé: gamète femelle servant à la reproduction sexuée; 2. Biol. corr. : O. dénoyauté: sac à mitochondries servant à réveiller la totipotentialité des noyaux somatiques diploïdes.

Parents. 1. Ring. : couple formé par un homme et une femme ayant engendré par reproduction sexuée. 2. Mod.: couple d'individus, associés dans le cadre d'une sentimental joint venture, et ayant mené à bien un projet de PTM. 
Père. Ring.: inutile depuis la PTM, puisque le noyau somatique peut aussi provenir de la «mère».

Procréation écologique. Ring. : enfantement à l'ancienne (v. PS).

Procréatique. Mod.: ensemble des procédures biologiquement de plus en plus correctes permettant à tout individu d'assurer sa descendance en l'affranchissant des contraintes physiologiques ou pathologiques.

Projet parental. 1. Ring.: désir d'un couple d'avoir un enfant par reproduction sexuée; 2. Mod.: désir de clone.

PS. Procréation sexuée. Ring., Biol. incorr.: ensemble des pratiques de reproduction en vigueur au temps de l'ère BD Abandonné au profit de la PTM. NB : ne donne plus droit à couverture sociale.

PTM. Procréation Totalement Maîtrisée. Biol. corr.: ensemble des procédures contrôlées de procréatique conduisant à l'obtention d'un individu conforme aux nouvelles normes Bio Iso 9001, comportant notamment une reproduction asexuée suivie de GEV. Degré le plus achevé de la correction biologique en matière de procréatique. A supplanté les autres techniques de PMA, telles que FIV, FIVETE, GIFT, ZIFT, IAD, ICSI etc. Seule procédure de procréatique remboursée par la Sécurité Sociale. La PTM a libéré l'homme et la femme de leur animalité.

Reproduction asexuée. Biol. corr. : stade ultime et complètement maîtrisé de la procréatique permettant de mener à bien un projet parental en s'affranchissant totalement des aléas de la loterie méiotique et des contraintes de l'animalité. Ce progrès a pu être accompli grâce au clonage (voir aussi clone, homoclone, autoclone, xénoclone, zyclone).

Reproduction sexuée. Ring.: mode de procréation in vivo soumis aux aléas de la loterie méiotique (voir PS).

Stérilité. Hist. : vaincue par la PTM. progrès biologique. Ils concernent certaines pratiques biologiques, révolutionnaires aussi longtemps qu'elles demeurent techniquement irréalisables. Ils cèdent si la pression sociale est trop forte. Les comités d'éthique permettent d'abord de les respecter, puis de les renverser (voir aussi Onzième commandement).

Transplantation nucléaire. Mod., Biol. corr. : premier stade de la PTM.

Xénoclone. Clone où le noyau donneur et l'ovocyte receveur ne sont pas de la même espèce.

Xénogestation. Implantation d'un zygote ou d'un homoclone dans un utérus de femelle d'une espèce différente. Désormais supplantée par la GEV.

Zyclone. Mod.: cellule obtenue au cours de la première étape du clonage d'individu. Formé par insertion d'un noyau somatique diploïde dans un ovocyte nulloploïde. Équivalent du zygote de la reproduction sexuée. Terme peu usité en raison des risques de confusion avec le gaz Zyklon.

\section{* ABRÉVIATIONS *}

Anim. animal: se refere à l'ensemble des pratiques de procréation biologiquement incorrectes en vigueur au temps où l'homme ne s'était pas encore affranchi des soi-disant "Lois de la Nature », c'est-à-dire de son animalité.

Arch. archaïque, se rapportant à des pratiques en usage au temps $d$ Homo sapiens sapiens.

Biol. corr. biologiquement correct.

Biol. incorr. biologiquement incorrect.

Hist. historique.

Mod. moderne, c'est-à-dire conforme aux idées à la mode.

Mythol. mythologie.

Philos. philosophie.

Procr. procréatique.

Ring. ringard: se rapportant à des pratiques révolues dans les pays biologiquement développés, mais encore en usage dans les populations non encore libérées des contraintes biologiques.

Soc. sociologie.

\section{Jean-Claude Kaplan}

Professeur de biochimie à la Faculté de médecine Cochin-Port-Royal. Service de biochimie et génétique moléculaire de l'Hôpital Cochin, Inserm U. 129, 24, rue du Faubourg-Saint-Jacques, 75014 Paris, France.

\section{TIRÉS À PART}

J.C. Kaplan.

\section{ROTARY INTERNATIONAL}

ASSOCIATION POUR LE DÉVELOPPEMENT DE LA RECHERCHE EN GÉRONTOLOGIE

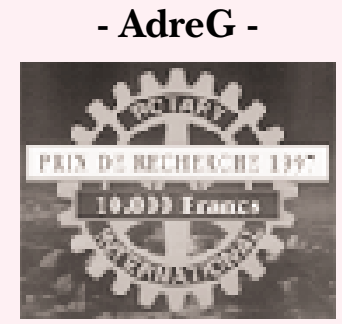

Ce prix s'adresse aux médecins, biologistes, psychologues..., ayant entrepris ou venant de terminer une recherche dans un domaine relevant de la gérontologie.

Pour postuler, il suffit d'envoyer un dossier comprenant une lettre de motivation, votre curriculum-vitae, ainsi qu'une copie de votre travail avant la fin mai 1997 en 3 exemplaires au:

$$
\text { Docteur de JAEGER }
$$

ASSOCIATION POUR LE DÉVELOPPEMENT DE LA RECHERCHE EN GÉRONTOLOGIE ROTARY CLUB DE MEUDON

37, avenue Galliéni - 92190 MEUDON

Un jury composé de scientifiques représentatifs étudiera les dossiers et attribuera le prix.

La remise des prix se fera fin 1997 en présence du Gouverneur du $1660^{\mathrm{e}}$ district, des rotariens et de la presse médicale.

Pour tout renseignement, contacter :

Mme BARON ou le Docteur de JAEGER Département de Gérontologie

Clinique Médicale du Château d'Herblay 50, rue de Paris, 95220 Herblay Tél. : 0134505014 